\title{
Final Results of GERDA on the Search for Neutrinoless Double- $\beta$ Decay
}

M. Agostini, ${ }^{9,17}$ G. R. Araujo, ${ }^{21}$ A. M. Bakalyarov, ${ }^{15}$ M. Balata, ${ }^{1}$ I. Barabanov, ${ }^{13}$ L. Baudis, ${ }^{21}$ C. Bauer, ${ }^{8}$ E. Bellotti, ${ }^{10,11}$ S. Belogurov ${ }^{14,13, \dagger}$ A. Bettini, ${ }^{18,19}$ L. Bezrukov, ${ }^{13}$ V. Biancacci, ${ }^{18,19}$ D. Borowicz, ${ }^{6}$ E. Bossio, ${ }^{17}$ V. Bothe, ${ }^{8}$ V. Brudanin, ${ }^{6}$ R. Brugnera, ${ }^{18,19}$ A. Caldwell, ${ }^{16}$ C. Cattadori, ${ }^{11}$ A. Chernogorov, ${ }^{14,15}$ T. Comellato, ${ }^{17}$ V. D' Andrea, ${ }^{2}$ E. V. Demidova, ${ }^{14}$ N. Di Marco, ${ }^{1}$ E. Doroshkevich, ${ }^{13}$ F. Fischer, ${ }^{16}$ M. Fomina, ${ }^{6}$ A. Gangapshev, ${ }^{13,8}$ A. Garfagnini, ${ }^{18,19}$ C. Gooch, ${ }^{16}$ P. Grabmayr, ${ }^{20}$ V. Gurentsov, ${ }^{13}$ K. Gusev, ${ }^{6,15}{ }^{17}$ J. Hakenmüller, ${ }^{8}$ S. Hemmer, ${ }^{19}$ R. Hiller, ${ }^{21}$ W. Hofmann, ${ }^{8}$ J. Huang, ${ }^{21}$ M. Hult, ${ }^{7}$ L. V. Inzhechik, ${ }^{13, \$}$ J. Janicskó Csáthy, ${ }^{17,}$ J. Jochum, ${ }^{20}$ M. Junker, ${ }^{1}$ V. Kazalov, ${ }^{13}$ Y. Kermaïdic, ${ }^{8}$ H. Khushbakht, ${ }^{20}$ T. Kihm, ${ }^{8}$ I. V. Kirpichnikov, ${ }^{14}$ A. Klimenko, ${ }^{8,6, \|}$ R. Kneiß1, ${ }^{16}$ K. T. Knöpfle $\odot,{ }^{8}$ O. Kochetov, ${ }^{6}$ V. N. Kornoukhov, ${ }^{13, \dagger}$ P. Krause, ${ }^{17}$ V. V. Kuzminov, ${ }^{13}$ M. Laubenstein, ${ }^{1}$ A. Lazzaro, ${ }^{17}$ M. Lindner, ${ }^{8}$ I. Lippi,${ }^{19}$ A. Lubashevskiy, ${ }^{6}$ B. Lubsandorzhiev, ${ }^{13}$ G. Lutter, ${ }^{7}$ C. Macolino, ${ }^{1,}$ B. Majorovits, ${ }^{16}$ W. Maneschg, ${ }^{8}$ L. Manzanillas, ${ }^{16}$ M. Miloradovic, ${ }^{21}$ R. Mingazheva, ${ }^{21}$ M. Misiaszek, ${ }^{4}$ P. Moseev, ${ }^{13}$ Y. Müller, ${ }^{21}$ I. Nemchenok, ${ }^{6, \|}$ K. Panas, ${ }^{4}$ L. Pandola, ${ }^{3}$ K. Pelczar, ${ }^{7}$ L. Pertoldi, ${ }^{18,19}$ P. Piseri, ${ }^{12}$ A. Pullia, ${ }^{12}$ C. Ransom, ${ }^{21}$ L. Rauscher, ${ }^{20}$ S. Riboldi, ${ }^{12}$ N. Rumyantseva, ${ }^{15,6}$ C. Sada ${ }^{18,19}$ F. Salamida, ${ }^{2}$ S. Schönert, ${ }^{17}$ J. Schreiner, ${ }^{8}$ M. Schütt, ${ }^{8}$ A.-K. Schütz,${ }^{20}$ O. Schulz,${ }^{16}$ M. Schwarz, ${ }^{17}$ B. Schwingenheuer, ${ }^{8}$ O. Selivanenko, ${ }^{13}$ E. Shevchik, ${ }^{6}$ M. Shirchenko, ${ }^{6}$ L. Shtembari, ${ }^{16}$ H. Simgen, ${ }^{8}$ A. Smolnikov, ${ }^{8,6}$ D. Stukov, ${ }^{15}$ A. A. Vasenko, ${ }^{14}$ A. Veresnikova, ${ }^{13}$ C. Vignoli, ${ }^{1}$ K. von Sturm, ${ }^{18,19}$ T. Wester, ${ }^{5}$ C. Wiesinger, ${ }^{17}$ M. Wojcik, ${ }^{4}$ E. Yanovich, ${ }^{13}$ B. Zatschler, ${ }^{5}$ I. Zhitnikov, ${ }^{6}$ S. V. Zhukov, ${ }^{15}$ D. Zinatulina, ${ }^{6}$ A. Zschocke, ${ }^{20}$ A. J. Zsigmond, ${ }^{16}$ K. Zuber, ${ }^{5}$ and G. Zuzel ${ }^{4}$

(GERDA Collaboration) ${ }^{*}$

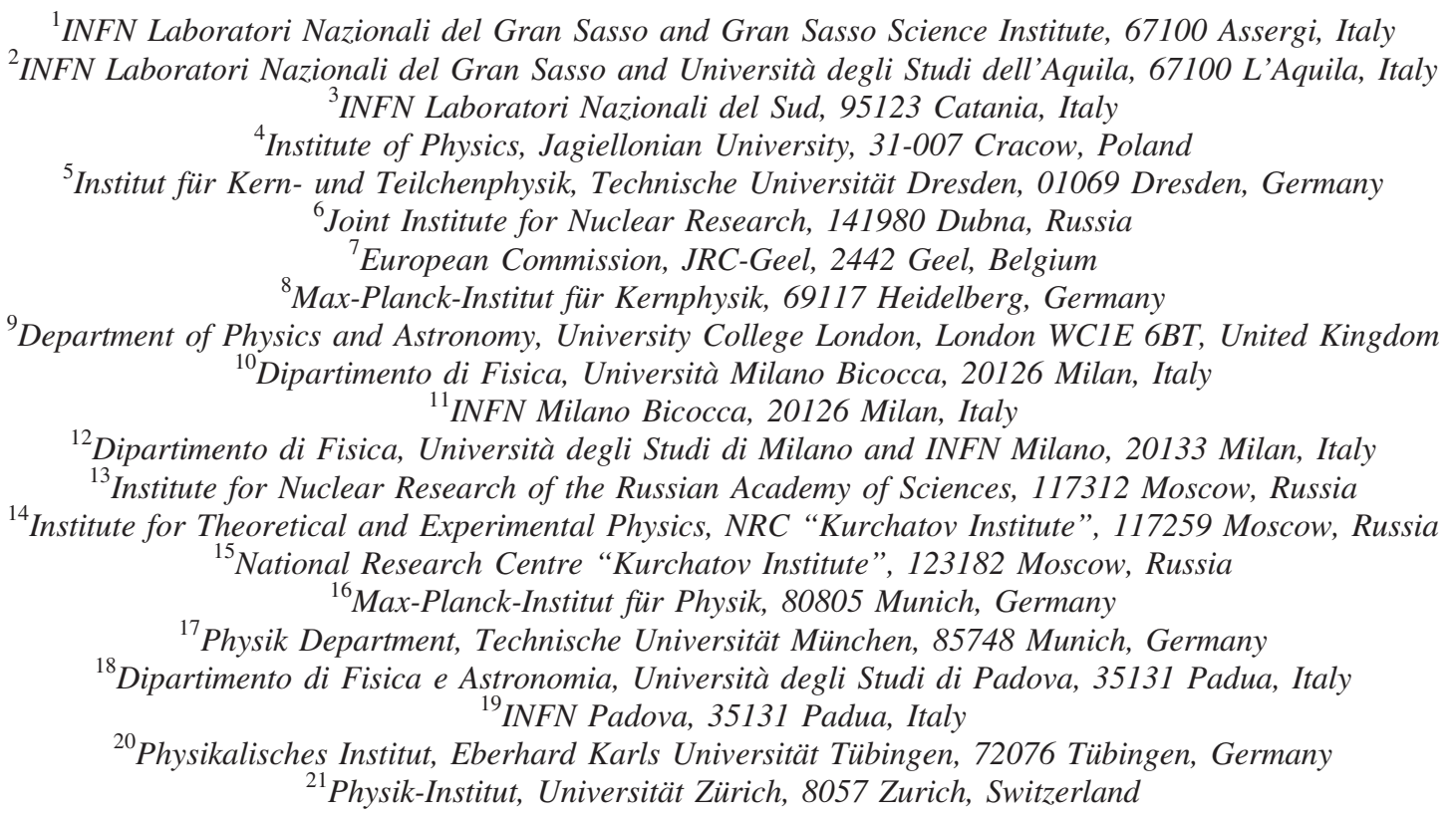

(Received 27 August 2020; revised 30 October 2020; accepted 11 November 2020; published 17 December 2020)

The GERmanium Detector Array (GERDA) experiment searched for the lepton-number-violating neutrinoless double- $\beta(0 \nu \beta \beta)$ decay of ${ }^{76} \mathrm{Ge}$, whose discovery would have far-reaching implications in cosmology and particle physics. By operating bare germanium diodes, enriched in ${ }^{76} \mathrm{Ge}$, in an active liquid argon shield, GERDA achieved an unprecedently low background index of $5.2 \times 10^{-4}$ counts/( $\mathrm{keV} \mathrm{kg} \mathrm{yr)}$ in the signal region and met the design goal to collect an exposure of $100 \mathrm{~kg}$ yr in a background-free

Published by the American Physical Society under the terms of the Creative Commons Attribution 4.0 International license. Further distribution of this work must maintain attribution to the author(s) and the published article's title, journal citation, and DOI. 
regime. When combined with the result of Phase I, no signal is observed after $127.2 \mathrm{~kg}$ yr of total exposure. A limit on the half-life of $0 \nu \beta \beta$ decay in ${ }^{76} \mathrm{Ge}$ is set at $T_{1 / 2}>1.8 \times 10^{26} \mathrm{yr}$ at $90 \%$ C.L., which coincides with the sensitivity assuming no signal.

DOI: 10.1103/PhysRevLett.125.252502

The matter-antimatter asymmetry of the Universe remains an important unsolved puzzle of cosmology and particle physics. Many theories predict that the asymmetry is produced by a violation of lepton number via leptogenesis [1]. These theories naturally lead to neutrinos being their own antiparticles and developing a Majorana mass component. Neutrino Majorana masses and lepton-number violation can be verified at the same time by observing a hypothetical nuclear transition $(A, Z) \rightarrow(A, Z+2)+2 e^{-}$, called neutrinoless double- $\beta(0 \nu \beta \beta)$ decay [2]. In $0 \nu \beta \beta$ decay, two neutrons in the parent nucleus convert into two protons and two electrons. Unlike the known neutrinoaccompanied double- $\beta(2 \nu \beta \beta)$ decay, the two electrons emitted in a $0 \nu \beta \beta$ decay would share the entire energy released in the process. The main experimental signature of $0 \nu \beta \beta$ decay is hence a characteristic peak in the energy distribution, located at the $Q$ value of the decay $\left(Q_{\beta \beta}\right)$. A vigorous experimental program is underway to search for this transition in various candidate isotopes: ${ }^{76} \mathrm{Ge}[3,4],{ }^{82} \mathrm{Se}$ [5], ${ }^{100} \mathrm{Mo}[6-8],{ }^{130} \mathrm{Te}[9,10],{ }^{136} \mathrm{Xe}[11-13]$, and others.

In this Letter, the final results of the GERmanium Detector Array (GERDA) experiment on the search for the $0 \nu \beta \beta$ decay of ${ }^{76} \mathrm{Ge}$ are presented. GERDA used highpurity germanium detectors made out of material isotopically enriched in ${ }^{76} \mathrm{Ge}$ to $\sim 87 \%$ [14,15]: this approach maximizes the detection efficiency as source and detector coincide. The outstanding energy resolution of germanium detectors guarantees a very clear signature of the $0 \nu \beta \beta$ decay signal. Background around $Q_{\beta \beta}=2039.06 \mathrm{keV}$ [16] was minimized by operating the bare detectors in liquid argon (LAr), which provides both shielding and cooling [17].

Phase I of GERDA collected $23.5 \mathrm{~kg}$ yr of exposure (= total germanium mass $\times$ live time) between November 2011 and September 2013, with an average background index $B$ of $11 \times 10^{-3}$ counts/(keVkgyr) at $Q_{\beta \beta}$ [18]. Phase II of GERDA started in December 2015, after a major upgrade [15] with additional germanium detectors of superior performance and a LAr veto system [19]. The goal was to reduce the background below $B=10^{-3}$ counts $/(\mathrm{keV} \mathrm{kg} \mathrm{yr})$ and to collect $100 \mathrm{~kg}$ yr of exposure in a background-free regime. In this regime the most probable number of background events in the signal region is zero and the sensitivity scales linearly with the exposure, instead of the square root. Initially, $20 \mathrm{~kg}$ of broad energy germanium (BEGe) detectors $[20,21]$ were added to $15.6 \mathrm{~kg}$ of coaxial detectors already operated in Phase I. After the last data release in 2018 [3], additional inverted coaxial (IC) detectors [22] with a total mass of $9.6 \mathrm{~kg}$ were installed, as summarized in Table I.

The GERDA experiment is located at the Laboratori Nazionali del Gran Sasso (LNGS) of INFN, Italy, where a rock overburden of $3500 \mathrm{~m}$ water equivalent reduces the flux from cosmic muons by 6 orders of magnitude. The array of germanium detectors is lowered in a cryostat containing $64 \mathrm{~m}^{3}$ of LAr through a lock system inside a clean room. The cryostat is surrounded by a water tank $\left(590 \mathrm{~m}^{3}\right.$ purified water) equipped with photomultipliers (PMTs) to detect the residual cosmic muons reaching the experiment. Water and LAr also shield the detector array from external natural radioactivity and neutrons. The muon

TABLE I. Summary of the GERDA Phase II parameters for different detector types and before and after the upgrade. The components of the total efficiency $\varepsilon$ for $0 \nu \beta \beta$ decays are reported individually. The efficiencies of muon veto and quality cuts are above $99.9 \%$ and are not shown. Energy resolutions and all $0 \nu \beta \beta$ decay detection efficiencies are reported as exposure-weighted averages for each detector type and their uncertainties are given as standard deviations.

\begin{tabular}{|c|c|c|c|c|c|}
\hline & \multicolumn{2}{|c|}{ Dec 2015-May 2018} & \multicolumn{3}{|c|}{ July 2018-Nov 2019} \\
\hline & Coaxial & BEGe & Coaxial & BEGe & Inverted coaxial \\
\hline Number of detectors & 7 & 30 & 6 & 30 & 5 \\
\hline Total mass & $15.6 \mathrm{~kg}$ & $20 \mathrm{~kg}$ & $14.6 \mathrm{~kg}$ & $20 \mathrm{~kg}$ & $9.6 \mathrm{~kg}$ \\
\hline Exposure $\mathcal{E}$ & $28.6 \mathrm{~kg} \mathrm{yr}$ & $31.5 \mathrm{~kg} \mathrm{yr}$ & $13.2 \mathrm{~kg}$ yr & $21.9 \mathrm{~kg} \mathrm{yr}$ & $8.5 \mathrm{~kg} \mathrm{yr}$ \\
\hline Energy resolution at $Q_{\beta \beta}(\mathrm{FWHM})$ & $(3.6 \pm 0.2) \mathrm{keV}$ & $(2.9 \pm 0.3) \mathrm{keV}$ & $(4.9 \pm 1.4) \mathrm{keV}$ & $(2.6 \pm 0.2) \mathrm{keV}$ & $(2.9 \pm 0.1) \mathrm{keV}$ \\
\hline $0 \nu \beta \beta$ decay detection efficiency $\varepsilon$ : & $(46.2 \pm 5.2) \%$ & $(60.5 \pm 3.3) \%$ & $(47.2 \pm 5.1) \%$ & $(61.1 \pm 3.9) \%$ & $(66.0 \pm 1.8) \%$ \\
\hline Electron containment & $(91.4 \pm 1.9) \%$ & $(89.7 \pm 0.5) \%$ & $(92.0 \pm 0.3) \%$ & $(89.3 \pm 0.6) \%$ & $(91.8 \pm 0.5) \%$ \\
\hline${ }^{76} \mathrm{Ge}$ enrichment & $(86.6 \pm 2.1) \%$ & $(88.0 \pm 1.3) \%$ & $(86.8 \pm 2.1) \%$ & $(88.0 \pm 1.3) \%$ & $(87.8 \pm 0.4) \%$ \\
\hline Active volume & $(86.1 \pm 5.8) \%$ & $(88.7 \pm 2.2) \%$ & $(87.1 \pm 5.8) \%$ & $(88.7 \pm 2.1) \%$ & $(92.7 \pm 1.2) \%$ \\
\hline Liquid argon veto & \multicolumn{2}{|c|}{$(97.7 \pm 0.1) \%$} & & $(98.2 \pm 0.1) \%$ & \\
\hline Pulse shape discrimination & $(69.1 \pm 5.6) \%$ & $(88.2 \pm 3.4) \%$ & $(68.8 \pm 4.1) \%$ & $(89.0 \pm 4.1) \%$ & $(90.0 \pm 1.8) \%$ \\
\hline
\end{tabular}


veto system [23] is complemented by scintillator panels installed on the top of the clean room.

The 41 germanium detectors are assembled into seven strings and each string is placed inside a nylon cylinder to limit the LAr volume from which radioactive ions can be collected by electric fields. This strategy effectively reduces the background due to the $\beta$ decay of ${ }^{42} \mathrm{~K}$, which is produced as a progeny of the long-lived ${ }^{42} \mathrm{Ar}$ and has a $Q$ value above $Q_{\beta \beta}$ [24].

A cylindrical volume around the array is instrumented with photosensors, which detect the scintillation light in the LAr. The LAr veto system consists of a curtain of wavelength-shifting fibers connected to silicon photomultipliers and 16 cryogenic PMTs [15,25]. During the upgrade, the geometrical coverage of the fiber curtain was improved.

The germanium detectors are connected to chargesensitive amplifiers located inside the LAr about $35 \mathrm{~cm}$ above the array. The signals are digitized at $25 \mathrm{MHz}$ for a total length of $160 \mu \mathrm{s}$ and at $100 \mathrm{MHz}$ in a $10-\mu \mathrm{s}$ window around the rising edge and are stored on disk for analysis.

The offline analysis of the digitized signals follows the procedures described in Ref. [26]. Since Phase I, the GERDA Collaboration adopted a strict blinded analysis: events with a reconstructed energy within $\pm 25 \mathrm{keV}$ of $Q_{\beta \beta}$ are removed from the data stream and not analyzed further until all analysis procedures and parameters have been finalized. The energy of the events in the germanium detectors is reconstructed with a zero-area cusp filter [27], whose parameters are optimized for each detector and calibration run. Weekly calibration runs with ${ }^{228} \mathrm{Th}$ sources are performed to determine the energy scale and resolution, as well as to define and monitor the analysis cuts. The energy resolutions, defined as full width at half maximum (FWHM), at $Q_{\beta \beta}$ of each detector type are summarized in Table I, together with their standard deviations. The new IC detectors show an average resolution of $2.9 \mathrm{keV}$, a remarkable achievement given their mass of $\sim 2 \mathrm{~kg}$, comparable to the coaxial detectors. In addition, they provide a similarly efficient identification of the event topology, and hence background rejection [28], as the much smaller $(\sim 0.7 \mathrm{~kg})$ BEGe detectors. The energy resolution is stable within $0.1 \mathrm{keV}$ for most of the detectors over the full data taking period. Gain stability and noise are monitored by test pulses injected into the front-end electronics at a rate of $0.05 \mathrm{~Hz}$. The fraction of data corresponding to stable operating conditions that are used for physics analysis is about $80 \%$ of the total. Signals originating from electrical discharges or bursts of noise are rejected by quality cuts based on the flatness of the baseline, polarity and time structure of the pulse. Physical events at $Q_{\beta \beta}$ are accepted with an efficiency larger than $99.9 \%$.

The two electrons emitted in a double- $\beta$ decay have a range in germanium of the order of $1 \mathrm{~mm}$ : they deposit their energy in a small volume of the detector and thus produce highly localized events (single-site events, SSEs). In contrast, $\gamma$ rays of similar energy mostly interact via Compton scattering and can produce events with several separated energy depositions (multiple-site events, MSEs). Events in which more than one germanium detector is fired are therefore identified as background. The unique feature in Phase II of GERDA is the LAr veto, that allows to reject events in which energy is deposited in the LAr volume surrounding the germanium detectors. If any of the photosensors detects a signal of at least one photoelectron within about $6 \mu \mathrm{s}$ of the germanium detector trigger, the event is classified as background. Accidental coincidences lead to a dead time of $(2.3 \pm 0.1) \%$ [(1.8 \pm 0.1$) \%]$ before (after) the upgrade, measured by randomly triggered events. Events are discarded also if preceded by a muon-veto signal within $10 \mu \mathrm{s}$; the induced dead time is $<0.01 \%$.

The pulse shape of the germanium detector signals is used to discriminate background events. In addition to $\gamma$ induced MSEs, events due to $\alpha$ or $\beta$ decays on the detector surface can also be identified. In the case of the BEGe and IC detectors one parameter, $A / E$, is used to classify background events, where $A$ is the maximum current amplitude and $E$ is the energy. As MSEs and surface events at the $n^{+}$electrode are characterized by wider current pulses, they feature a lower $A / E$ value compared to SSEs, while surface events at the very thin $(<1 \mu \mathrm{m}) p^{+}$ electrode show a higher $A / E$ value [29]. Therefore, rejecting events on both sides of the $A / E$ distribution of SSEs enhances the signal to background ratio. The coaxial detectors feature a more complicated time structure which requires an artificial neural network (ANN) to discriminate SSEs from MSEs and a dedicated cut on the signal rise time to discard events on the $p^{+}$electrode $[3,30]$.

An additional cut is applied to all detectors to remove events with slow or incomplete charge collection [3]. These events are not necessarily due to background but rather to energy depositions in particular parts of the detectors featuring unusual charge collection dynamics. These events are identified through the difference between two energy estimates performed using the same digital filter but different shaping times. An event is discarded if the energy difference is larger than 3 standard deviations from the average.

${ }^{228} \mathrm{Th}$ calibration data are used to train the ANN and to tune the $A / E$ discrimination. The double escape peak (DEP) at $1593 \mathrm{keV}$ of the prominent $\gamma$ ray of ${ }^{208} \mathrm{Tl}$ at $2615 \mathrm{keV}$ is used as a sample of SSEs, and the full energy peak at $1621 \mathrm{keV}$ from ${ }^{212} \mathrm{Bi}$ as a sample of MSEs. The MSE cut threshold is set for all detectors at $90 \%$ DEP survival fraction. The threshold to reject $p^{+}$surface events is optimized using the $2 \nu \beta \beta$ and $\alpha$ decays. The $0 \nu \beta \beta$ decay signal efficiency is estimated for all detectors from the survival fraction of DEP and $2 \nu \beta \beta$ decay events after all cuts. An extrapolation to $Q_{\beta \beta}$ is performed to take into account the energy dependence. The combined signal efficiency of pulse shape discrimination is reported in Table I for each detector type, before and after the upgrade. 


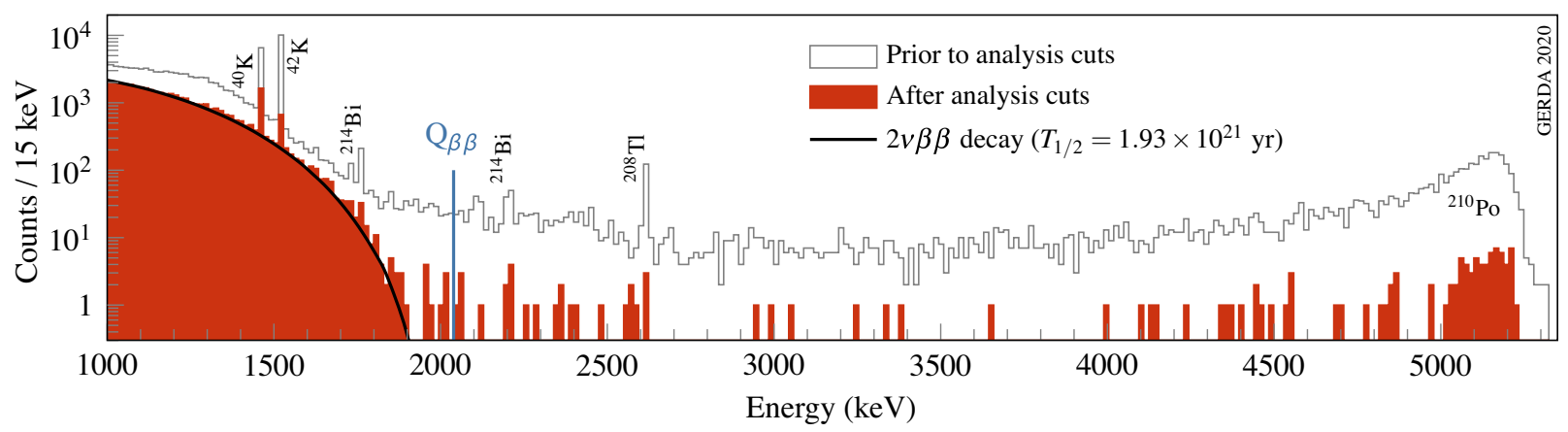

FIG. 1. Energy distribution of GERDA Phase II events between 1.0 and $5.3 \mathrm{MeV}$ before and after analysis cuts; the exposure is $103.7 \mathrm{~kg}$ yr. The expected distribution of $2 \nu \beta \beta$ decay events is shown assuming the half-life measured by GERDA [31]. The prominent $\gamma$ lines and the $\alpha$ population around 5.2 $\mathrm{MeV}$ are also labeled.

GERDA Phase II data were collected between December 2015 and November 2019. The total exposure is $103.7 \mathrm{~kg}$ yr (58.9 kg yr already published in Ref. [3] and $44.8 \mathrm{~kg} \mathrm{yr}$ of new data). Figure 1 shows the energy distribution of all events before and after applying the analysis cuts. At low energy, the counting rate is mostly accounted for by the $2 \nu \beta \beta$ decay of ${ }^{76} \mathrm{Ge}$ with a half-life of $T_{1 / 2}^{2 \nu \beta \beta}=(1.926 \pm 0.094) \times 10^{21} \mathrm{yr}[31]$.

The energy range considered for the $0 \nu \beta \beta$ decay analysis goes from 1930 to $2190 \mathrm{keV}$, with the exclusion of the intervals $(2104 \pm 5)$ and $(2119 \pm 5) \mathrm{keV}$ that contain two known background peaks (Fig. 2). No other $\gamma$ line or structure is expected in this analysis window according to the background model [32]. After unblinding, 13 events are found in this analysis window after all cuts (5 in coaxial, 7 in BEGe and 1 in IC detectors). These events are likely due to $\alpha$ decays, ${ }^{42} \mathrm{~K} \beta$ decays, or $\gamma$ decays from ${ }^{238} \mathrm{U}$ and ${ }^{232} \mathrm{Th}$ series. Coaxial detector data which were unblinded in Ref. [33], when less effective PSD techniques against surface events were available, and which were also included in the analysis in Refs. [3,34], have been reanalyzed according to the new method. As a consequence, three events—at energies 1968, 2061, and $2064 \mathrm{keV}$ - that were previously included in the analysis window are now discarded.

The energy distribution of the events in the analysis window is fitted to search for a signal due to $0 \nu \beta \beta$ decay. The fit model includes a Gaussian distribution for the signal, centered at $Q_{\beta \beta}$ with a width corresponding to the energy resolution, and a flat distribution for the background. The free parameters of the fit are the signal strength $S=1 / T_{1 / 2}$ and the background index $B$. The expectation value of the number of signal events scales with $S$ as

$$
\mu_{s}=\frac{\ln 2 \mathcal{N}_{A}}{m_{76}} \varepsilon \mathcal{E} S,
$$

where $\mathcal{N}_{A}$ is Avogadro's number, $m_{76}$ the molar mass of ${ }^{76} \mathrm{Ge}, \mathcal{E}$ the exposure, and $\varepsilon$ the total efficiency of detecting $0 \nu \beta \beta$ decays. The average $0 \nu \beta \beta$ decay detection efficiency of each detector type and its breakdown in individual components are listed in Table I. The mean number of background events in the analysis window is given by

$$
\mu_{b}=B \times \Delta E \times \mathcal{E},
$$

with $\Delta E=240 \mathrm{keV}$ being the net width of the analysis window. Data of each detector are divided in partitions, i.e., periods of time in which parameters are stable. Each partition $k$ is characterized by its own energy resolution

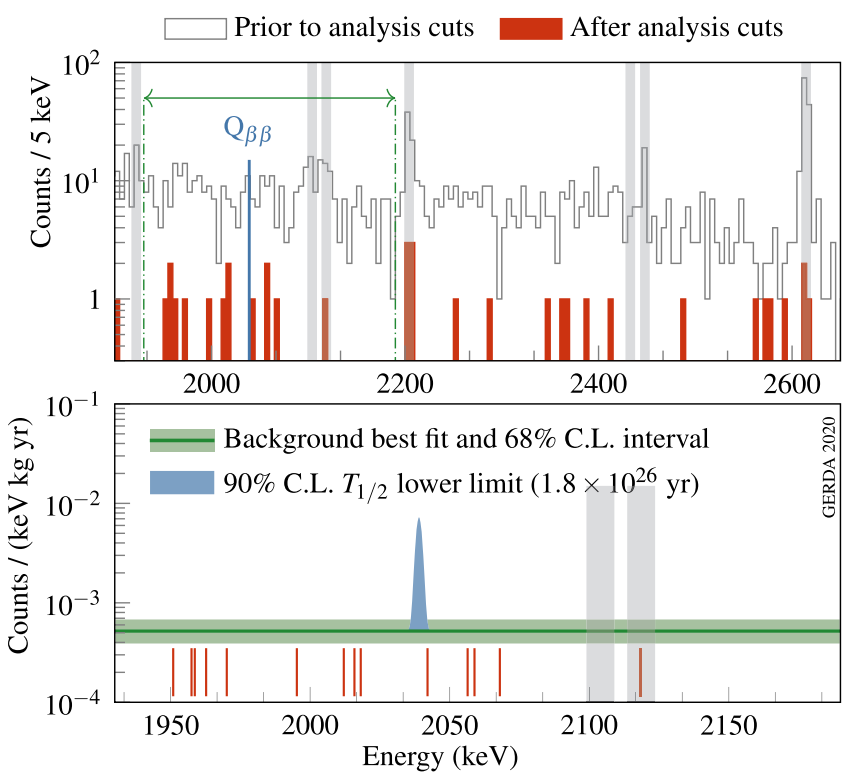

FIG. 2. Top: Enlarged view of the energy distribution of GERDA Phase II events between 1900 and $2650 \mathrm{keV}$ before and after analysis cuts. This energy interval includes the analysis window (edges marked by dashed lines) and the regions of expected $\gamma$ lines (marked by gray areas), among those the prominent $\gamma$ line at $2615 \mathrm{keV}$. Bottom: Result of the unbinned extended likelihood fit: The blue peak displays the expected $0 \nu \beta \beta$ decay signal for $T_{1 / 2}$ equal to the lower limit, $1.8 \times 10^{26} \mathrm{yr}$. Its width is the resolution $\sigma_{k}$ of the partition which contains the event closest to $Q_{\beta \beta}$. Vertical lines indicate the energies of the events in the analysis window after analysis cuts. 
$\sigma_{k}=\mathrm{FWHM} / 2.35$, efficiency $\varepsilon_{k}$ and exposure $\mathcal{E}_{k}$. The signal strength $S$ and the background index $B$ instead are common parameters to all partitions. This construction is a significant improvement compared to the analysis used in the past $[3,33,34]$ as it allows a precise tracing of the performance of each detector at any given moment. Furthermore, the background index is now assumed to be the same for all detectors, while independent parameters for each detector type were used previously. This change is motivated by the lack of any statistically significant indication of a different background depending on detector type, position within the array, or time.

The statistical analysis is based on an unbinned extended likelihood function and it is performed in both frequentist and Bayesian frameworks, following the procedure described in Ref. [33]. The likelihood function is given by the product of likelihoods of each partition, weighted with the Poisson term:

$$
\begin{aligned}
\mathcal{L}= & \prod_{k}\left[\frac{\left(\mu_{s, k}+\mu_{b, k}\right)^{N_{k}} e^{-\left(\mu_{s, k}+\mu_{b, k}\right)}}{N_{k} !} \times\right. \\
& \left.\prod_{i=1}^{N_{k}} \frac{1}{\mu_{s, k}+\mu_{b, k}} \times\left(\frac{\mu_{b, k}}{\Delta E}+\frac{\mu_{s, k}}{\sqrt{2 \pi} \sigma_{k}} e^{-\frac{\left(E_{i}-Q_{\beta \beta}\right)^{2}}{2 \sigma_{k}^{2}}}\right)\right],
\end{aligned}
$$

where $E_{i}$ is the energy of the $N_{k}$ events in the $k$ th partition. The parameters $\mu_{s, k}$ and $\mu_{b, k}$ are calculated from Eqs. (1) and (2) and are partition dependent. Phase I datasets are included in the analysis as individual partitions with independent background indices.

The frequentist analysis is performed using a two-sided test statistics based on the profile likelihood. The probability distributions of the test statistic are computed using Monte Carlo techniques, as they are found to significantly deviate from $\chi^{2}$ distributions. The analysis of the $N=13$ events of Phase II yields no indication for a signal and a lower limit of $T_{1 / 2}>1.5 \times 10^{26} \mathrm{yr}$ at $90 \%$ C.L. is set. Phase I and Phase II data together give a total exposure of $127.2 \mathrm{~kg}$ yr, which corresponds to $(1.288 \pm 0.018) \mathrm{kmol} \mathrm{yr}$ of ${ }^{76} \mathrm{Ge}$ in the active volume. The combined analysis has also a best fit for null signal strength, and provides a halflife limit of

$$
T_{1 / 2}>1.8 \times 10^{26} \mathrm{yr} \text { at } 90 \% \text { C.L. }
$$

The limit coincides with the sensitivity, defined as the median expectation under the no signal hypothesis.

GERDA achieved an unprecedentedly low background in Phase II, as derived from the fit, of $B=5.2_{-1.3}^{+1.6} \times$ $10^{-4}$ counts/( $\left.\mathrm{keV} \mathrm{kg} \mathrm{yr}\right)$, and met the design goal of background-free performance: the mean background expected in the signal region $\left(Q_{\beta \beta} \pm 2 \sigma\right)$ is 0.3 counts.

The statistical analysis is carried out also within a Bayesian framework. The one-dimensional posterior probability density function $P(S \mid$ data $)$ of the signal strength is derived by

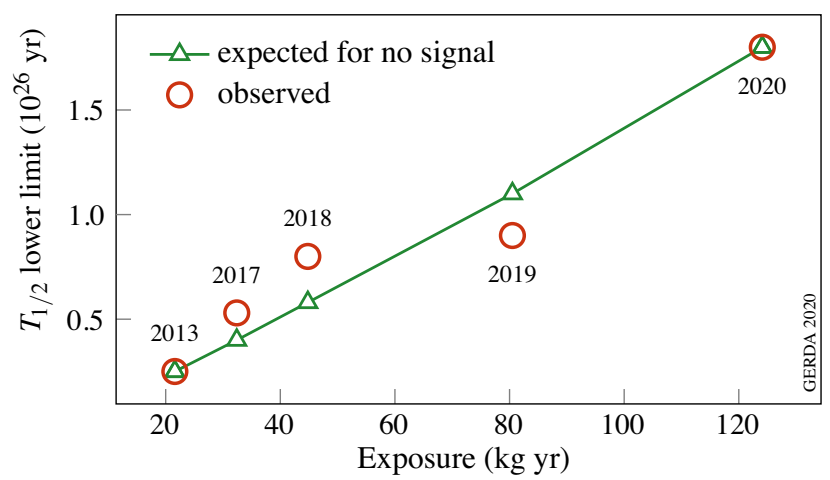

FIG. 3. Circles: lower limit (90\% C.L.) on the $0 \nu \beta \beta$ decay halflife of ${ }^{76} \mathrm{Ge}$ set by GERDA as a function of the exposure $[3,18,33,34]$. Triangles: median expectation in the assumption of no signal.

marginalizing over the other free parameters by using the Bayesian analysis toolkit BAT [35]. The prior distribution for $S$ is assumed to be constant between 0 and $10^{-24} 1 / \mathrm{yr}$, as in previous GERDA works. The limit on the half-life from Phases I and II together is $T_{1 / 2}>1.4 \times 10^{26} \mathrm{yr}$ (90\% C.I.). A stronger limit $2.3 \times 10^{26} \mathrm{yr}(90 \%$ C.I. $)$ is obtained assuming a priori equiprobable Majorana neutrino masses $m_{\beta \beta}$ (as $S \propto m_{\beta \beta}^{2}$ ), instead of equiprobable signal strengths.

Uncertainties on the energy reconstruction, energy resolution, and efficiencies are folded into the analysis through additional nuisance parameters, each constrained by a Gaussian probability distribution. Their overall effect on the limit is at the percent level. Potential systematic uncertainties related to the fit model are found to marginally impact the results. For instance, the limit changes by a few percent if a linear energy distribution is assumed for the background.

Figure 3 shows the improvement achieved by GERDA with increasing exposure for the measured lower limit on the $0 \nu \beta \beta$ decay half-life of ${ }^{76} \mathrm{Ge}$ and for the sensitivity. The background-free regime results in a nearly linear improvement of sensitivity vs exposure. GERDA is the experiment providing the best sensitivity and the most stringent constraint on the half-life of any $0 \nu \beta \beta$ decay.

The $T_{1 / 2}$ limit can be converted into an upper limit on the effective Majorana neutrino mass under the assumption that the decay is dominated by the exchange of light Majorana neutrinos. Assuming a standard value of $g_{A}=1.27$, the phase space factor and the set of nuclear matrix elements from Refs. [36-46], a limit of $m_{\beta \beta}<79-180 \mathrm{meV}$ at 90\% C.L. is obtained, which is comparable to the most stringent constraints from other isotopes $[9,11,12]$.

GERDA has been a pioneering experiment in the search for $0 \nu \beta \beta$ decay. GERDA improved the sensitivity by one order of magnitude with respect to previous ${ }^{76} \mathrm{Ge}$ experiments $[47,48]$ and proved that a background-free experiment based on ${ }^{76} \mathrm{Ge}$ is feasible. Indeed, the LEGEND Collaboration [49] is preparing a next generation 
experiment with a sensitivity to the half-life of $0 \nu \beta \beta$ decay up to $10^{28} \mathrm{yr}$ and beyond. In the first phase, LEGEND-200 has taken over the GERDA infrastructure at LNGS and will start data taking in 2021.

The data shown in Figs. 1 and 3 and the data relevant for the GERDA Phase II statistical analysis are available in ASCII format as Supplemental Material [50].

The GERDA experiment is supported financially by the German Federal Ministry for Education and Research (BMBF), the German Research Foundation (DFG), the Italian Istituto Nazionale di Fisica Nucleare (INFN), the Max Planck Society (MPG), the Polish National Science Centre (NCN), the Foundation for Polish Science (TEAM/ 2016-2/17), the Russian Foundation for Basic Research, and the Swiss National Science Foundation (SNF). This project has received funding and support from the European Union's Horizon 2020 research and innovation programme under the Marie Sklodowska-Curie Grant Agreements No. 690575 and No. 674896. The institutions acknowledge also internal financial support. The GERDA Collaboration thanks the directors and the staff of the LNGS for their continuous strong support of the GERDA experiment.

*gerda-eb@mpi-hd.mpg.de

${ }^{\dagger}$ Also at NRNU MEPhI, 115409 Moscow, Russia.

${ }^{*}$ Also at Moscow Institute of Physics and Technology, 117303 Moscow, Russia.

${ }^{\S}$ Present address: Leibniz-Institut für Kristallzüchtung, 12489 Berlin, Germany.

"Also at Dubna State University, 141982 Dubna, Russia.

"Present address: LAL, CNRS/IN2P3, Université ParisSaclay, 91405 Orsay, France.

[1] S. Davidson, E. Nardi, and Y. Nir, Leptogenesis, Phys. Rep. 466, 105 (2008).

[2] J. Schechter and J. W. F. Valle, Neutrinoless double beta decay in $\mathrm{SU}(2) \times \mathrm{U}(1)$ theories, Phys. Rev. D 25, 2951 (1982).

[3] M. Agostini et al. (GERDA Collaboration), Probing Majorana neutrinos with double- $\beta$ decay, Science 365, 1445 (2019).

[4] S. I. Alvis et al. (MAJORANA Collaboration), Search for neutrinoless double- $\beta$ decay in ${ }^{76} \mathrm{Ge}$ with $26 \mathrm{~kg}$-yr of exposure from the MAJORANA DEMONSTRATOR, Phys. Rev. C 100, 025501 (2019).

[5] O. Azzolini et al. (CUPID Collaboration), Final Result of CUPID-0 Phase-I in the Search for the ${ }^{82} \mathrm{Se}$ Neutrinoless Double- $\beta$ Decay, Phys. Rev. Lett. 123, 032501 (2019).

[6] V. Alenkov et al. (AMoRE Collaboration), First results from the AMoRE-Pilot neutrinoless double beta decay experiment, Eur. Phys. J. C 79, 791 (2019).

[7] R. Arnold et al. (NEMO-3 Collaboration), Results of the search for neutrinoless double- $\beta$ decay in ${ }^{100} \mathrm{Mo}$ with the NEMO-3 experiment, Phys. Rev. D 92, 072011 (2015).
[8] E. Armengaud et al., The CUPID-Mo experiment for neutrinoless double-beta decay: performance and prospects, Eur. Phys. J. C 80, 44 (2020).

[9] D. Q. Adams et al. (CUORE Collaboration), Improved Limit on Neutrinoless Double-Beta Decay in ${ }^{130} \mathrm{Te}$ with CUORE, Phys. Rev. Lett. 124, 122501 (2020).

[10] S. Andringa et al. (SNO+ Collaboration), Current status and future prospects of the SNO + Experiment, Adv. High Energy Phys. 2016, 6194250 (2016).

[11] G. Anton et al. (EXO-200 Collaboration), Search for Neutrinoless Double- $\beta$ Decay with the Complete EXO200 Dataset, Phys. Rev. Lett. 123, 161802 (2019).

[12] A. Gando et al. (KamLAND-Zen Collaboration), Search for Majorana Neutrinos Near the Inverted Mass Hierarchy Region with KamLAND-Zen, Phys. Rev. Lett. 117, 082503 (2016).

[13] J. Martín-Albo et al. (NEXT Collaboration), Sensitivity of NEXT-100 to neutrinoless double beta decay, J. High Energy Phys. 05 (2016) 159.

[14] K.-H. Ackermann et al. (GERDA Collaboration), The GERDA experiment for the search of $0 \nu \beta \beta$ decay in ${ }^{76} \mathrm{Ge}$, Eur. Phys. J. C 73, 2330 (2013).

[15] M. Agostini et al. (GERDA Collaboration), Upgrade for Phase II of the GERDA experiment, Eur. Phys. J. C 78, 388 (2018).

[16] B. J. Mount, M. Redshaw, and E. G. Myers, Double- $\beta$-decay $Q$ values of ${ }^{74} \mathrm{Se}$ and ${ }^{76} \mathrm{Ge}$, Phys. Rev. C 81, 032501(R) (2010).

[17] G. Heusser, Low-radioactivity background techniques, Annu. Rev. Nucl. Part. Sci. 45, 543 (1995).

[18] M. Agostini et al. (GERDA Collaboration), Results on Neutrinoless Double- $\beta$ Decay of ${ }^{76} \mathrm{Ge}$ from Phase I of the GERDA Experiment, Phys. Rev. Lett. 111, 122503 (2013).

[19] M. Agostini et al., LArGe: active background suppression using argon scintillation for the GERDA $0 \nu \beta \beta$-experiment, Eur. Phys. J. C 75, 506 (2015).

[20] M. Agostini et al. (GERDA Collaboration), Production, characterization and operation of ${ }^{76} \mathrm{Ge}$ enriched BEGe detectors in GERDA, Eur. Phys. J. C 75, 39 (2015).

[21] M. Agostini et al. (GERDA Collaboration), Characterization of $30{ }^{76} \mathrm{Ge}$ enriched Broad Energy Ge detectors for GERDA Phase II, Eur. Phys. J. C 79, 978 (2019).

[22] R. J. Cooper, D. C. Radford, P. A. Hausladen, and K. Lagergren, A novel HPGe detector for gamma-ray tracking and imaging, Nucl. Instrum. Methods Phys. Res., Sect. A 665, 25 (2011).

[23] K. Freund et al., The performance of the Muon Veto of the GERDA Experiment, Eur. Phys. J. C 76, 298 (2016).

[24] A. Lubashevskiy et al., Mitigation of ${ }^{42} \mathrm{Ar} /{ }^{42} \mathrm{~K}$ background for the GERDA Phase II experiment, Eur. Phys. J. C 78, 15 (2018).

[25] J. Janicskó Csáthy, H. Aghaei Khozani, A. Caldwell, X. Liu, and B. Majorovits, Development of an anti-Compton veto for HPGe detectors operated in liquid argon using silicon photo-multipliers, Nucl. Instrum. Methods Phys. Res., Sect. A 654, 225 (2011).

[26] M. Agostini, L. Pandola, and P. Zavarise, Off-line data processing and analysis for the GERDA experiment, J. Phys. Conf. Ser. 368, 012047 (2012). 
[27] M. Agostini et al. (GERDA Collaboration), Improvement of the energy resolution via an optimized digital signal processing in GERDA Phase I, Eur. Phys. J. C 75, 255 (2015).

[28] A. Domula, M. Hult, Y. Kermaïdic, G. Marissens, B. Schwingenheuer, T. Wester, and K. Zuber, Pulse shape discrimination performance of inverted coaxial Ge detectors, Nucl. Instrum. Methods Phys. Res., Sect. A 891, 106 (2018).

[29] D. Budjáš, M. Barnabé Heider, O. Chkvorets, N. Khanbekov, and S. Schönert, Pulse shape discrimination studies with a Broad-Energy Germanium detector for signal identification and background suppression in the GERDA double beta decay experiment, J. Instrum. 4, P10007 (2009).

[30] M. Agostini et al., Pulse shape discrimination for GERDA Phase I data, Eur. Phys. J. C 73, 2583 (2013).

[31] M. Agostini et al. (GERDA Collaboration), Results on $\beta \beta$ decay with emission of two neutrinos or Majorons in ${ }^{76} \mathrm{Ge}$ from GERDA Phase I, Eur. Phys. J. C 75, 416 (2015).

[32] M. Agostini et al. (GERDA Collaboration), Modeling of GERDA Phase II data, J. High Energy Phys. 03 (2020) 139.

[33] M. Agostini et al. (GERDA Collaboration), Backgroundfree search for neutrinoless double- $\beta$ decay of ${ }^{76} \mathrm{Ge}$ with GERDA, Nature 544, 47 (2017).

[34] M. Agostini et al. (GERDA Collaboration), Improved Limit on Neutrinoless Double- $\beta$ Decay of ${ }^{76} \mathrm{Ge}$ from GERDA Phase II, Phys. Rev. Lett. 120, 132503 (2018).

[35] O. Schulz, F. Beaujean, A. Caldwell, C. Grunwald, V. Hafych, K. Kröninger, S. La Cagnina, L. Röhrig, and L. Shtembari, BAT.jl-A Julia-based tool for Bayesian inference, arXiv:2008.03132.

[36] T. R. Rodríguez and G. Martínez-Pinedo, Energy Density Functional Study of Nuclear Matrix Elements for Neutrinoless $\beta \beta$ Decay, Phys. Rev. Lett. 105, 252503 (2010).

[37] M. T. Mustonen and J. Engel, Large-scale calculations of the double- $\beta$ decay of ${ }^{76} \mathrm{Ge},{ }^{130} \mathrm{Te},{ }^{136} \mathrm{Xe}$, and ${ }^{150} \mathrm{Nd}$ in the deformed self-consistent Skyrme quasiparticle randomphase approximation, Phys. Rev. C 87, 064302 (2013).

[38] N. L. Vaquero, T. R. Rodríguez, and J. L. Egido, Shape and Pairing Fluctuation Effects on Neutrinoless Double Beta Decay Nuclear Matrix Elements, Phys. Rev. Lett. 111, 142501 (2013).
[39] M. Horoi and A. Neacsu, Shell model predictions for ${ }^{124}$ Sn double- $\beta$ decay, Phys. Rev. C 93, 024308 (2016).

[40] J. Hyvärinen and J. Suhonen, Nuclear matrix elements for $0 \nu \beta \beta$ decays with light or heavy Majorana-neutrino exchange, Phys. Rev. C 91, 024613 (2015).

[41] J. Barea, J. Kotila, and F. Iachello, $0 \nu \beta \beta$ and $2 \nu \beta \beta$ nuclear matrix elements in the interacting boson model with isospin restoration, Phys. Rev. C 91, 034304 (2015).

[42] J. Menéndez, Neutrinoless $\beta \beta$ decay mediated by the exchange of light and heavy neutrinos: the role of nuclear structure correlations, J. Phys. G 45, 014003 (2018).

[43] L. S. Song, J. M. Yao, P. Ring, and J. Meng, Nuclear matrix element of neutrinoless double- $\beta$ decay: Relativity and short-range correlations, Phys. Rev. C 95, 024305 (2017).

[44] F. Šimkovic, A. Smetana, and P. Vogel, $0 \nu \beta \beta$ and $2 \nu \beta \beta$ nuclear matrix elements evaluated in closure approximation, neutrino potentials and SU(4) symmetry, Phys. Rev. C 98, 064325 (2018)

[45] D.-L. Fang, A. Faessler, and F. Šimkovic, $0 \nu \beta \beta$-decay nuclear matrix element for light and heavy neutrino mass mechanisms from deformed quasiparticle random-phase approximation calculations for ${ }^{76} \mathrm{Ge},{ }^{82} \mathrm{Se},{ }^{130} \mathrm{Te},{ }^{136} \mathrm{Xe}$, and ${ }^{150} \mathrm{Nd}$ with isospin restoration, Phys. Rev. C 97, 045503 (2018).

[46] L. Coraggio, A. Gargano, N. Itaco, R. Mancino, and F. Nowacki, The calculation of the neutrinoless double- $\beta$ decay matrix element within the realistic shell model, Phys. Rev. C 101, 044315 (2020).

[47] H. V. Klapdor-Kleingrothaus et al., Latest results from the HEIDELBERG-MOSCOW double beta decay experiment, Eur. Phys. J. A 12, 147 (2001).

[48] C. E. Aalseth et al., Recent results of the IGEX ${ }^{76} \mathrm{Ge}$ double-beta decay experiment, Phys. At. Nucl. 63, 1225 (2000).

[49] N. Abgrall et al. (LEGEND Collaboration), The large enriched germanium experiment for neutrinoless double beta decay (LEGEND), AIP Conf. Proc. 1894, 020027 (2017).

[50] See Supplemental Material at http://link.aps.org/ supplemental/10.1103/PhysRevLett.125.252502 for the data shown in Figs. 1 and 3 and the data for the GERDA Phase II statistical analysis. 